\title{
THE PAN-PACIFIC PLANET SEARCH. I. A GIANT PLANET ORBITING 7 CMa
}

\author{
Robert A. Wittenmyer ${ }^{1}$, Michael Endi ${ }^{2}$, Liang WAng ${ }^{3}$, John Asher Johnson ${ }^{4}$, C. G. Tinney ${ }^{1}$, And S. J. O’Toole ${ }^{5}$ \\ ${ }^{1}$ Department of Astrophysics, School of Physics, University of New South Wales, NSW 2052, Australia; rob@ phys.unsw.edu.au \\ ${ }^{2}$ McDonald Observatory, University of Texas at Austin, 1 University Station C1400, Austin, TX 78712, USA \\ ${ }^{3}$ Key Laboratory of Optical Astronomy, National Astronomical Observatories, Chinese Academy of Sciences, \\ A20 Datun Road, Chaoyang District, Beijing 100012, China \\ ${ }^{4}$ Department of Astrophysics, California Institute of Technology, MC 249-17, Pasadena, CA 91125, USA \\ ${ }^{5}$ Australian Astronomical Observatory, P.O. Box 296, Epping, NSW 1710, Australia \\ Received 2011 August 30; accepted 2011 November 3; published 2011 December 2
}

\begin{abstract}
We introduce the Pan-Pacific Planet Search, a survey of 170 metal-rich Southern Hemisphere subgiants using the $3.9 \mathrm{~m}$ Anglo-Australian Telescope. We report the first discovery from this program, a giant planet orbiting $7 \mathrm{CMa}$ (HD 47205) with a period of $763 \pm 17$ days, eccentricity $e=0.14 \pm 0.06$, and $m \sin i=2.6 \pm 0.6 M_{\text {Jup }}$. The host star is a $K$ giant with a mass of $1.5 \pm 0.3 M_{\odot}$ and metallicity $[\mathrm{Fe} / \mathrm{H}]=0.21 \pm 0.10$. The mass and period of $7 \mathrm{CMa} b$ are typical of planets which have been found to orbit intermediate-mass stars $\left(M_{*}>1.3 M_{\odot}\right)$. Hipparcos photometry shows this star to be stable to 0.0004 mag on the radial-velocity period, giving confidence that this signal can be attributed to reflex motion caused by an orbiting planet.
\end{abstract}

Key words: planetary systems - stars: individual (HD 47205) - techniques: radial velocities

Online-only material: color figure, machine-readable table

\section{INTRODUCTION}

Nearly 20 years of concerted radial-velocity monitoring of solar-type main-sequence stars has unveiled a fascinating diversity of planets and planetary system configurations. From the many hundreds of planets now characterized, the observational evidence is mounting for several interesting relationships between the properties of planets and their host stars. Among these are (1) giant planet occurrence is positively correlated with stellar metallicity (Fischer \& Valenti 2005) and mass (Johnson et al. 2010a; Endl et al. 2006), (2) short-period "SuperEarths" with $m \sin i<10 M_{\oplus}$ are about an order of magnitude more common than close-in giant planets (Howard et al. 2010; Wittenmyer et al. 2011), and (3) planet mass is positively correlated with host star mass (Bowler et al. 2010).

Most of the stars which have been targeted by radialvelocity surveys have masses which fall in the range $0.7-1.3 M_{\odot}$ (Johnson 2008; Valenti \& Fischer 2005). This is a consequence of the technical requirements of Doppler exoplanetary detection, which demand that stars be cool enough to present an abundance of spectral lines, and rotate slowly enough that their absorption lines are not significantly broadened by rotation. Stars of lower mass (e.g., $M$ dwarfs) are intrinsically faint in the optical, making the acquisition of high signal-to-noise spectra extremely expensive in telescope time (Endl et al. 2006). Main-sequence stars of higher mass have few usable absorption lines (due to their high temperatures) and also tend to be fast rotators ( $v \sin i>50 \mathrm{~km} \mathrm{~s}^{-1}$; Galland et al. 2005) due to their youth. In addition, the shorter main-sequence lifetimes of higher-mass stars mean that they will preferentially be observed at younger ages. Stars earlier than about F7 also have much shallower convection zones and so do not experience the magnetic braking which slows the rotation of later-type (lower-mass) stars. As a result, only the most massive planets can be detected orbiting $A$ and $F$ dwarfs. It is only recently that a significant number of planetary systems have been discovered orbiting intermediatemass stars $\left(M_{*}>1.3 M_{\odot}\right)$. These stars have proven to be a fertile hunting ground for interesting planetary systems, such as the 4:3 mean-motion resonant planets orbiting HD 200964 (Johnson et al. 2011). Now, some headway is beginning to be made in addressing the crucial question of how planet formation depends on stellar mass (Bowler et al. 2010; Johnson et al. 2010a; Sato et al. 2010). A number of surveys are seeking to expand our knowledge of planetary systems orbiting stars more massive than the Sun (e.g., Setiawan et al. 2003; Hatzes et al. 2005; Sato et al. 2005; Johnson et al. 2006b; Döllinger et al. 2007; Niedzielski et al. 2009). These surveys are exploiting the advantage wrought by stellar evolution: as stars evolve off the main sequence into subgiants and giants, their atmospheres expand and cool, making precision Doppler velocity measurements possible due to an abundance of narrow spectral lines.

The well-known planet-metallicity correlation (Gonzalez 1999; Fischer \& Valenti 2005), whereby main-sequence stars with higher metal content are more likely to host planets, has come under some scrutiny. Analysis of the metallicities of planet-hosting giant stars by Schuler et al. (2005) showed that the giant star planet hosts were significantly more metal-poor than their main-sequence counterparts. Pasquini et al. (2007) have also argued that the planet-metallicity correlation does not apply for evolved stars. They propose that this is evidence for a "pollution" scenario, in which main-sequence stars hosting planets appear metal-rich because they have accreted material from the protoplanetary disk (Murray \& Chaboyer 2002). When a star evolves off the main sequence, the convective zone increases in size by about a factor of 35 (Pasquini et al. 2007). If the high metallicities observed in planet hosts are due to pollution, this expansion of the convective zone will significantly dilute the extent of that pollution, and the subgiant's photosphere would return to its "birth" metallicity. Hence, one would not expect a significant correlation between metallicity and planet frequency for subgiants.

However, the importance of planet pollution was downplayed even by the authors who proposed it, as they felt it should play only a minor role in shaping the planet-metallicity correlation seen in dwarf stars. Further, Valenti \& Fischer (2008), 
Johnson et al. 2010a, Takeda et al. (2007), and others found no evidence of a decreasing planet-metallicity correlation among $F$ dwarfs, subgiants, or $K$ giants. The sample of $K$ giants studied by Pasquini et al. (2007) had a limited metallicity range, with $[\mathrm{Fe} / \mathrm{H}]<+0.2$. Examination of the form of the planet-metallicity correlation of Fischer \& Valenti (2005) and Johnson et al. (2010a) shows that, for small numbers of stars, the correlation over this metallicity range would look approximately flat.

One way to test this is to search for planets around a sample of evolved stars that are unambiguously metal-rich. Sandage et al. (2003) pointed out that stars on the red-edge of the subgiant branch represent such a population. Here, we introduce a new Southern Hemisphere survey, the "Pan-Pacific Planet Search (PPPS)," which uses the $3.9 \mathrm{~m}$ Anglo-Australian Telescope (AAT) to search for planets among these evolved, metal-rich stars to test for evidence of planet pollution.

In this paper, we present the first result from this new survey: the detection of a $2.6 M_{\text {Jup }}$ planet orbiting the nearby evolved star HD 47205. In Section 2, we introduce the PPPS and give a complete target list for the survey. Section 3 describes the observational data and gives the stellar parameters for $7 \mathrm{CMa}$. In Section 4, we detail the orbit-fitting process and present the planetary parameters. Finally, in Section 5 we discuss the further implications of this discovery.

\section{THE PAN-PACIFIC PLANET SEARCH}

\subsection{Survey Strategy and Target Selection}

The PPPS originated as a Southern Hemisphere extension of the established Lick \& Keck Observatory survey for planets orbiting Northern "retired A stars" (Johnson et al. 2006b, 2007, 2010a). This program is using the $3.9 \mathrm{~m}$ AAT to observe a metal-rich sample of Southern Hemisphere subgiants. We have selected 170 Southern stars with the following criteria: $1.0<(B-V)<1.2,1.8<M_{V}<3.0$, and $V<8.0$. By requiring $(B-V)>1$, we extend the red limit of the Johnson et al. (2006b) survey to the colors that stellar models indicate will be dominated by metal-rich subgiants (Girardi et al. 2002). This aims to deliver improved planetary detection statistics at $[\mathrm{Fe} / \mathrm{H}]>0.0$. In light of the observed positive correlation between stellar metallicity and planet occurrence, this should also deliver a roughly equivalent number of planetary detections to that obtained at Lick and Keck, though for metal-rich hosts. At the same time, by requiring $M_{V}>1.8$, we exclude giantbranch stars, as these have significant intrinsic velocity noise ("jitter") due to random convective motion and pulsations (Saar et al. 1998; Wright 2005)—typically about $20 \mathrm{~m} \mathrm{~s}^{-1}$ (Hekker et al. 2006). Our target list includes about 30 stars from the Lick survey; this overlap will serve as a check on the systematics between the two telescopes. Together, the three telescopes are observing more than 600 stars. The complete PPPS target list is given in Table 1.

Observing time is scheduled such that each target should receive 4-6 observations per year. This strategy would appear to reduce the probability of detecting shorter-period planets ( $P \lesssim 50$ days), which require more densely sampled observations in continuous blocks of time (O'Toole et al. 2009a, 2009b; Vogt et al. 2010). However, we note that the same scheduling has been used in the Lick and Keck survey, which has detected the $P=6.5$ day planet orbiting HD 102956 (Johnson et al. 2010b). By employing this strategy, we are able to target more stars with a fixed amount of observing time, which should increase the
Table 1

Pan-Pacific Planet Search Target List

\begin{tabular}{|c|c|c|c|}
\hline Star & R.A. & Decl. & $V$ \\
\hline 224910 & 000144.93 & -163154.2 & 7.83 \\
\hline 749 & 001138.06 & -493921.2 & 7.91 \\
\hline 1817 & 002221.22 & -505933.4 & 6.68 \\
\hline 2643 & 002954.99 & -321623.8 & 8.15 \\
\hline 4145 & 004350.09 & -120040.7 & 6.01 \\
\hline 5676 & 005812.43 & -255235.8 & 7.89 \\
\hline 5877 & 005919.25 & -582417.2 & 7.78 \\
\hline 6037 & 010138.60 & -161555.3 & 6.47 \\
\hline 7931 & 011832.20 & -284358.7 & 7.89 \\
\hline 9218 & 013013.84 & -285155.5 & 7.96 \\
\hline 9925 & 013543.11 & -531159.8 & 7.82 \\
\hline 10731 & 014328.25 & -561404.1 & 7.97 \\
\hline 11343 & 015006.22 & -542753.5 & 7.88 \\
\hline 11653 & 015300.51 & -524130.1 & 7.91 \\
\hline 12974 & 020656.07 & -014925.2 & 7.49 \\
\hline 13471 & 021054.48 & -320342.9 & 7.65 \\
\hline 13652 & 021234.49 & -261920.7 & 7.92 \\
\hline 14805 & 022043.08 & -623245.8 & 7.68 \\
\hline 14791 & 022207.00 & -360623.8 & 7.87 \\
\hline 15414 & 022612.48 & -625505.2 & 7.92 \\
\hline 19810 & 031051.47 & -110729.4 & 7.22 \\
\hline 20035 & 031157.63 & -392157.1 & 6.98 \\
\hline 20924 & 032158.79 & -152731.4 & 7.26 \\
\hline 24316 & 035132.65 & -170958.8 & 7.71 \\
\hline 25069 & 035852.42 & -052810.3 & 5.85 \\
\hline 28901 & 043206.76 & -284822.0 & 7.42 \\
\hline 29399 & 043334.10 & -624925.1 & 5.79 \\
\hline 31860 & 045746.42 & -345332.3 & 7.60 \\
\hline 34851 & 051202.21 & -752137.6 & 7.85 \\
\hline 33844 & 051236.08 & -145704.3 & 7.29 \\
\hline 37763 & 053152.66 & -762030.0 & 5.18 \\
\hline 39281 & 054834.16 & -534034.1 & 7.85 \\
\hline 40409 & 055405.90 & -630527.7 & 4.65 \\
\hline 43429 & 061517.71 & -182837.2 & 5.99 \\
\hline 46262 & 062053.87 & -790400.5 & 7.31 \\
\hline 47141 & 063605.42 & -245157.8 & 7.45 \\
\hline 47205 & 063641.00 & -191520.6 & 3.95 \\
\hline 51268 & 065333.56 & -545259.3 & 7.97 \\
\hline 58540 & 072257.03 & -553438.8 & 6.89 \\
\hline 59663 & 072509.11 & -702413.9 & 7.75 \\
\hline 67644 & 080620.28 & -540245.9 & 7.97 \\
\hline 72467 & 083201.89 & -292215.1 & 7.59 \\
\hline 76321 & 085457.73 & -154645.9 & 7.10 \\
\hline 76437 & 085501.65 & -340835.0 & 7.15 \\
\hline 76920 & 085516.78 & -671555.9 & 7.83 \\
\hline 80275 & 091746.62 & -354123.8 & 7.70 \\
\hline 81410 & 092449.04 & -234934.4 & 7.35 \\
\hline 84070 & 094113.31 & -462255.1 & 7.88 \\
\hline 85128 & 094301.74 & -793530.2 & 7.30 \\
\hline 85035 & 094847.03 & -191848.6 & 7.02 \\
\hline 87089 & 100034.00 & -614531.3 & 7.93 \\
\hline 86950 & 100137.61 & -171958.8 & 7.47 \\
\hline HIP50638 & 102033.31 & -233825.4 & 7.54 \\
\hline 94386 & 105332.86 & -152644.5 & 6.34 \\
\hline 98516 & 111947.64 & -281119.6 & 7.06 \\
\hline 98579 & 112019.05 & -281956.1 & 6.68 \\
\hline 100939 & 113648.17 & -370220.5 & 7.94 \\
\hline 104358 & 120100.72 & -262847.2 & 7.76 \\
\hline 104704 & 120322.27 & -551917.0 & 7.49 \\
\hline 104819 & 120411.05 & -222215.6 & 7.93 \\
\hline 105096 & 120601.36 & -541528.1 & 7.03 \\
\hline 108991 & 123138.47 & -305854.8 & 6.73 \\
\hline 109866 & 123849.98 & -620154.1 & 7.76 \\
\hline 110238 & 124059.85 & -314415.9 & 7.70 \\
\hline 114899 & 131426.28 & -545743.6 & 7.99 \\
\hline 115066 & 131504.35 & -301053.0 & 7.83 \\
\hline
\end{tabular}


Table 1

(Continued)

\begin{tabular}{|c|c|c|c|}
\hline Star & R.A. & Decl. & $V$ \\
\hline 115202 & 131558.58 & -195634.2 & 5.21 \\
\hline 121056 & 135352.27 & -351851.1 & 6.17 \\
\hline 121156 & 135416.75 & -283409.9 & 6.05 \\
\hline 121930 & 135946.00 & -501340.5 & 7.58 \\
\hline 124087 & 141145.85 & -190103.2 & 7.74 \\
\hline 125774 & 142149.23 & -104000.5 & 7.99 \\
\hline 126105 & 142400.33 & -194802.8 & 7.32 \\
\hline 130048 & 144613.51 & -074748.9 & 7.14 \\
\hline 131182 & 145221.07 & -112822.7 & 7.95 \\
\hline 132396 & 150000.35 & -360149.7 & 6.94 \\
\hline 133166 & 150436.20 & -435350.4 & 7.92 \\
\hline 133670 & 150627.10 & -220154.1 & 6.13 \\
\hline 134443 & 151131.92 & -451644.7 & 7.38 \\
\hline 134692 & 151459.90 & -665336.5 & 7.91 \\
\hline 135760 & 151817.43 & -412513.0 & 7.05 \\
\hline 136295 & 152041.44 & -255924.0 & 7.11 \\
\hline 136905 & 152326.06 & -063636.7 & 7.29 \\
\hline 137115 & 152457.58 & -220237.1 & 7.65 \\
\hline 137164 & 152745.75 & -630114.1 & 7.44 \\
\hline 136135 & 152747.09 & -791822.4 & 7.61 \\
\hline 138061 & 152959.95 & -124635.6 & 7.78 \\
\hline 138716 & 153410.52 & -100350.3 & 4.61 \\
\hline 138973 & 153608.23 & -214446.6 & 7.72 \\
\hline 142132 & 155424.55 & -411022.3 & 7.70 \\
\hline 142384 & 155556.46 & -404711.0 & 7.41 \\
\hline 143561 & 160245.22 & -423025.5 & 7.97 \\
\hline 144073 & 160501.36 & -265652.0 & 7.60 \\
\hline 145428 & 161151.34 & -255300.3 & 7.73 \\
\hline 148760 & 163122.87 & -263215.2 & 6.07 \\
\hline 153438 & 170029.72 & -212741.3 & 7.35 \\
\hline 153937 & 170611.92 & -602514.8 & 7.43 \\
\hline 154250 & 170640.99 & -480043.5 & 7.96 \\
\hline 155233 & 171104.37 & -203915.2 & 6.81 \\
\hline 154556 & 171219.85 & -704315.2 & 6.21 \\
\hline 159743 & 173701.69 & -185930.9 & 7.45 \\
\hline 162030 & 174957.49 & -241225.1 & 7.02 \\
\hline 166309 & 181115.84 & -293822.4 & 7.61 \\
\hline 166476 & 181420.10 & -584220.9 & 7.81 \\
\hline 170707 & 183333.37 & -501241.5 & 7.75 \\
\hline 170286 & 183502.98 & -711926.1 & 7.72 \\
\hline 173902 & 184917.14 & -344456.0 & 6.59 \\
\hline 175905 & 185735.98 & -003134.6 & 7.66 \\
\hline 176002 & 190001.36 & -432049.7 & 7.92 \\
\hline 175304 & 190329.12 & -760654.8 & 7.75 \\
\hline 177897 & 190842.79 & -450434.3 & 7.74 \\
\hline 176794 & 191044.78 & -762415.7 & 6.94 \\
\hline 181342 & 192104.26 & -233710.2 & 7.55 \\
\hline 181809 & 192240.30 & -203833.6 & 6.72 \\
\hline 188981 & 195856.37 & -303217.7 & 6.27 \\
\hline 191067 & 200801.75 & -004040.9 & 5.97 \\
\hline 196676 & 203905.86 & -045546.2 & 6.46 \\
\hline 199809 & 210019.00 & -272035.9 & 7.93 \\
\hline 200073 & 210227.05 & -383150.0 & 5.93 \\
\hline 201931 & 211416.67 & -454657.2 & 6.89 \\
\hline 204073 & 212625.07 & -120542.0 & 6.70 \\
\hline 204057 & 212627.06 & -151442.9 & 7.97 \\
\hline 204203 & 212729.20 & -201245.2 & 7.84 \\
\hline 205577 & 213643.65 & -213009.8 & 7.93 \\
\hline 205972 & 213915.19 & -135341.0 & 7.25 \\
\hline 205478 & 214128.47 & -772322.1 & 3.73 \\
\hline 208431 & 215647.43 & -284903.3 & 7.91 \\
\hline 208791 & 215901.31 & -111703.4 & 7.79 \\
\hline 214573 & 224007.11 & -493553.2 & 7.37 \\
\hline 215005 & 224245.92 & -372043.7 & 7.93 \\
\hline 216640 & 225445.60 & -161618.3 & 5.53 \\
\hline 216643 & 225511.14 & -464043.9 & 7.53 \\
\hline
\end{tabular}

Table 1

(Continued)

\begin{tabular}{lccc}
\hline \hline Star & R.A. & Decl. & $V$ \\
\hline 218266 & 230711.97 & -455033.2 & 7.92 \\
219553 & 231649.69 & -211210.7 & 7.25 \\
222076 & 233808.10 & -705412.3 & 7.47 \\
222768 & 234332.71 & -225407.7 & 7.81 \\
223301 & 234828.19 & -113031.4 & 7.60 \\
223860 & 235313.59 & -110052.6 & 7.66 \\
\hline
\end{tabular}

(This table is also available in a machine-readable form in the online journal.)

probability of detecting the types of planets which are known to orbit nearly $20 \%$ of these types of stars (Johnson et al. 2010a).

\subsection{Observations and Data Reduction}

PPPS Doppler measurements are made with the UCLES echelle spectrograph (Diego et al. 1991) at the $3.9 \mathrm{~m}$ AAT. UCLES achieves a resolution of 45,000 with a 1 arcsec slit. An iodine absorption cell provides wavelength calibration from 5000 to $6200 \AA$. The spectrograph point-spread function and wavelength calibration are derived from the iodine absorption lines embedded on every pixel of the spectrum by the cell (Valenti et al. 1995; Butler et al. 1996). The result is a precision Doppler velocity estimate for each epoch, along with an internal uncertainty estimate, which includes the effects of photoncounting uncertainties, residual errors in the spectrograph PSF model, and variation in the underlying spectrum between the iodine-free template and epoch spectra observed through the iodine cell. The photon-weighted mid-time of each exposure is determined by an exposure meter. All velocities are measured relative to the zero point defined by the template observation. Velocities are obtained using the Austral code as first discussed in Endl et al. (2000). Austral is a proven Doppler code which has been used by the McDonald Observatory planet-search programs for nearly 10 years (e.g., Endl et al. 2004, 2006; Wittenmyer et al. 2009).

Observations for the PPPS began at the AAT in 2009 February. Since its inception, the program has received 20 nights per year, of which approximately $50 \%$ have resulted in usable data. We aim for a signal-to-noise ratio $(\mathrm{S} / \mathrm{N})$ of 100 at $5500 \AA$ per spectral pixel each epoch, resulting in exposure times ranging from $100 \mathrm{~s}$ up to a maximum of 20 minutes.

We have observed $7 \mathrm{CMa}$ on 21 epochs, and an iodine-free template observation was obtained on 2010 January 30. Since $7 \mathrm{CMa}$ is an extremely bright star, exposure times ranged from 100 to $500 \mathrm{~s}$, with a resulting $\mathrm{S} / \mathrm{N}$ of $\sim 200-300$ pixel $^{-1}$ each epoch. The data span a total of 917 days and have a mean internal velocity uncertainty of $6.5 \mathrm{~m} \mathrm{~s}^{-1}$.

\section{STELLAR PARAMETERS OF 7 CMa}

$7 \mathrm{CMa}$ (=HD 47205, HIP 31592) is one of the brightest stars in the PPPS survey $(V=3.95)$. In addition, it is accessible from most sites in both hemispheres (R.A.: 0636 41.038, decl.: -19 15 21.17), and so it has been well studied. Table 2 summarizes the physical parameters of this star. We have used our iodine-free template spectrum to derive spectroscopic stellar parameters, using methods described fully in Wang et al. (2011). In brief, $7 \mathrm{CMa}$ is an evolved, somewhat metal-rich $([\mathrm{Fe} / \mathrm{H}] \sim 0.2)$, intermediate-mass $\operatorname{star}\left(1.52 M_{\odot}\right)$ with a low level of activity. Hipparcos observations indicate that it is photometrically stable, with a median Hipparcos magnitude of $4.1200 \pm 0.0004$ (van Leeuwen 2007; Perryman et al. 1997). 
Table 2

Stellar Parameters for $7 \mathrm{CMa}$

\begin{tabular}{|c|c|c|}
\hline Parameter & Value & Reference \\
\hline Spec. type & K1 III & Gray et al. (2006) \\
\hline$M_{V}$ & $2.46 \pm 0.03$ & da Silva et al. (2006) \\
\hline$B-V$ & $1.037 \pm 0.041$ & van Leeuwen (2007) \\
\hline \multirow[t]{2}{*}{ Mass $\left(M_{\odot}\right)$} & $1.52 \pm 0.30$ & This work \\
\hline & $1.32 \pm 0.12$ & da Silva et al. (2006) \\
\hline Radius $\left(R_{\odot}\right)$ & $2.3 \pm 0.1$ & This work \\
\hline Luminosity $\left(L_{\odot}\right)$ & $11.3 \pm 0.3$ & This work \\
\hline Distance (pc) & $19.75 \pm 0.09$ & van Leeuwen (2007) \\
\hline \multirow[t]{2}{*}{$v \sin i\left(\mathrm{~km} \mathrm{~s}^{-1}\right)$} & 1.15 & Hekker \& Meléndez (2007) \\
\hline & 1.0 & Massarotti et al. (2008) \\
\hline$S_{\mathrm{MW}^{\mathrm{a}}}$ & 0.132 & Gray et al. (2006) \\
\hline \multirow[t]{3}{*}[\mathrm{Fe}/\mathrm{H}]{} & $0.21 \pm 0.10$ & This work \\
\hline & $0.18 \pm 0.1$ & da Silva et al. (2006) \\
\hline & 0.21 & Hekker \& Meléndez (2007) \\
\hline \multirow[t]{4}{*}{$v_{\text {micro }}\left(\mathrm{km} \mathrm{s}^{-1}\right)$} & $1.32 \pm 0.10$ & This work \\
\hline & 1.30 & da Silva et al. (2006) \\
\hline & 1.45 & Hekker \& Meléndez (2007) \\
\hline & 1.0 & Gray et al. (2006) \\
\hline \multirow[t]{4}{*}{$T_{\text {eff }}(\mathrm{K})$} & $4792 \pm 100$ & This work \\
\hline & $4744 \pm 70$ & da Silva et al. (2006) \\
\hline & 4830 & Hekker \& Meléndez (2007) \\
\hline & 4799 & Gray et al. (2006) \\
\hline \multirow[t]{4}{*}{$\log g$} & $3.25 \pm 0.10$ & This work \\
\hline & $3.11 \pm 0.07$ & da Silva et al. (2006) \\
\hline & 3.40 & Hekker \& Meléndez (2007) \\
\hline & 3.05 & Gray et al. (2006) \\
\hline
\end{tabular}

Note. ${ }^{a}$ Mount Wilson $S$-index.

\section{ORBIT FITTING AND PLANETARY PARAMETERS}

The AAT data show a root-mean-square (rms) scatter of $29.5 \mathrm{~m} \mathrm{~s}^{-1}$ about the mean velocity. Visual investigation of the data after two years of observation revealed a clear sinusoidal trend. Due to the relative paucity of data points $(N=21)$ compared to typical radial-velocity planet detections $(N \gtrsim 40)$, the traditional periodogram approach does not produce reliable estimates of statistical significance. Rather, since the periodic signal is readily apparent by eye, we used a genetic algorithm (Charbonneau 1995) to determine Keplerian orbital parameters. Those parameters were then used as initial inputs for a standard least-squares fitting routine. Our previous experience with genetic algorithms (Cochran et al. 2007; Tinney et al. 2011) has shown that the solution "evolves" quite rapidly toward a sharp $\chi^{2}$ minimum when brought to bear on data containing a real and coherent Keplerian signal. Indeed, with an allowed period range of 600-800 days, the algorithm converged on a solution with a period of 769 days and a small eccentricity $e=0.23$. We then used the GaussFit code (Jefferys et al. 1987) to obtain a Keplerian model fit for the planet. For the final orbit fitting, we added $5 \mathrm{~m} \mathrm{~s}^{-1}$ of jitter in quadrature to the internal uncertainties of the data shown in Table 3. The jitter estimate of $5 \mathrm{~m} \mathrm{~s}^{-1}$ is derived from Johnson et al. (2010a). In that work, 382 velocity measurements from 72 stable stars in the Lick and Keck survey of "retired A stars" were used to make an empirical jitter estimate which was then applied to the seven planet-host stars described therein. It would be ideal to estimate jitter for PPPS stars using the same methodology, but at this time, we have insufficient data on radial-velocity stable stars to make a statistically meaningful estimate. This is due to the short time baseline ( 2.5 years) and limited available data on a smaller

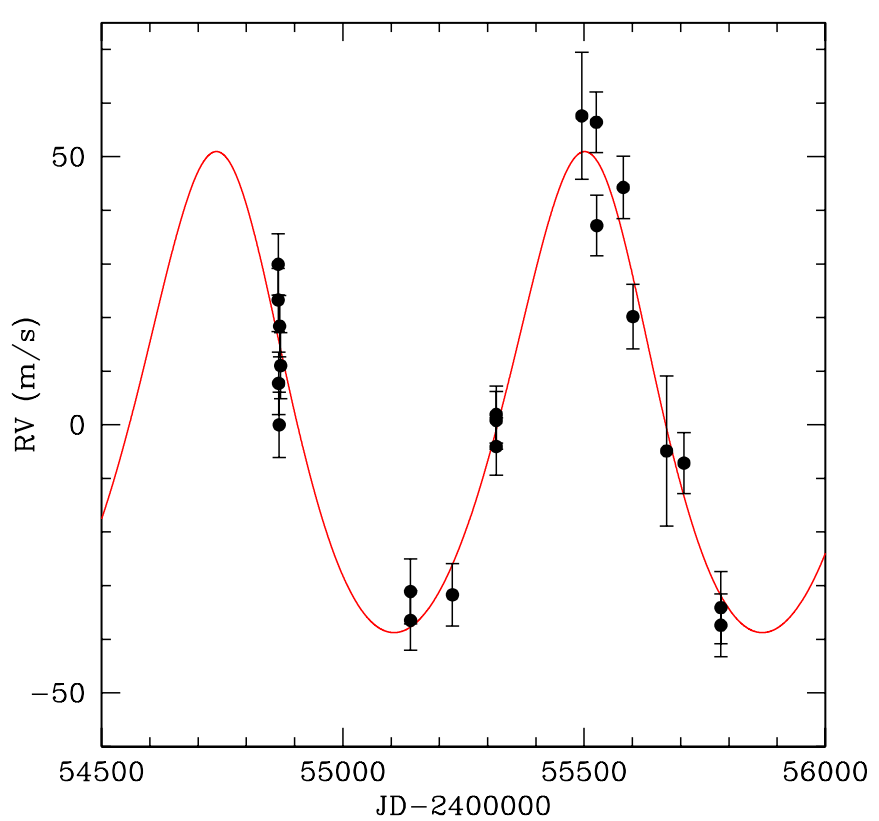

Figure 1. Radial-velocity data and Keplerian orbit fit for $7 \mathrm{CMa}$. The rms scatter about the fit is $7.5 \mathrm{~m} \mathrm{~s}^{-1}$, consistent with the mean uncertainty of $8.3 \mathrm{~m} \mathrm{~s}^{-1}$ (including $5 \mathrm{~m} \mathrm{~s}^{-1}$ of jitter added in quadrature).

(A color version of this figure is available in the online journal.)

Table 3

AAT Radial Velocities for $7 \mathrm{CMa}$

\begin{tabular}{lrr}
\hline \hline JD-2400000 & $\begin{array}{r}\text { Velocity } \\
\left(\mathrm{m} \mathrm{s}^{-1}\right)\end{array}$ & $\begin{array}{r}\text { Uncertainty } \\
\left(\mathrm{m} \mathrm{s}^{-1}\right)\end{array}$ \\
\hline 54866.09800 & 24.11 & 5.73 \\
54866.10107 & 17.47 & 5.86 \\
54866.94000 & 1.93 & 5.81 \\
54867.91576 & -5.81 & 6.09 \\
54869.08575 & 12.59 & 5.72 \\
54871.03478 & 5.22 & 6.13 \\
55140.18702 & -42.28 & 5.56 \\
55140.19229 & -36.90 & 6.07 \\
55227.06602 & -37.51 & 5.82 \\
55317.85529 & -4.99 & 5.40 \\
55317.85839 & -9.87 & 5.33 \\
55317.86143 & -3.89 & 5.31 \\
55495.13719 & 51.81 & 11.84 \\
55525.22369 & 50.62 & 5.68 \\
55526.21028 & 31.35 & 5.66 \\
55581.09317 & 38.45 & 5.83 \\
55601.00002 & 14.37 & 6.01 \\
55670.87749 & -10.70 & 13.99 \\
55706.84304 & -12.95 & 5.68 \\
55783.30394 & -43.20 & 5.85 \\
55783.31112 & -39.91 & 6.72 \\
\hline
\end{tabular}

number of stars in the PPPS as compared to the well-established Lick \& Keck survey. However, we consider the $5 \mathrm{~m} \mathrm{~s}^{-1}$ jitter estimate to be a reasonable approximation for PPPS targets due to the similarity in physical properties to the Johnson et al. (2010a) sample. We also note that there is substantial ( $\gtrsim 50 \%)$ uncertainty in the estimation of radial-velocity jitter (Wright 2005).

Using a stellar mass of $1.52 \pm 0.30 M_{\odot}$, we estimate the minimum mass $m \sin i$ to be $2.6 \pm 0.6 M_{\text {Jup }}$. The fit is shown in Figure 1 and the planetary parameters are given in Table 4. The residuals of the fit show no evidence for additional signals (Figure 2). As a further test of the veracity of the planet fit, 


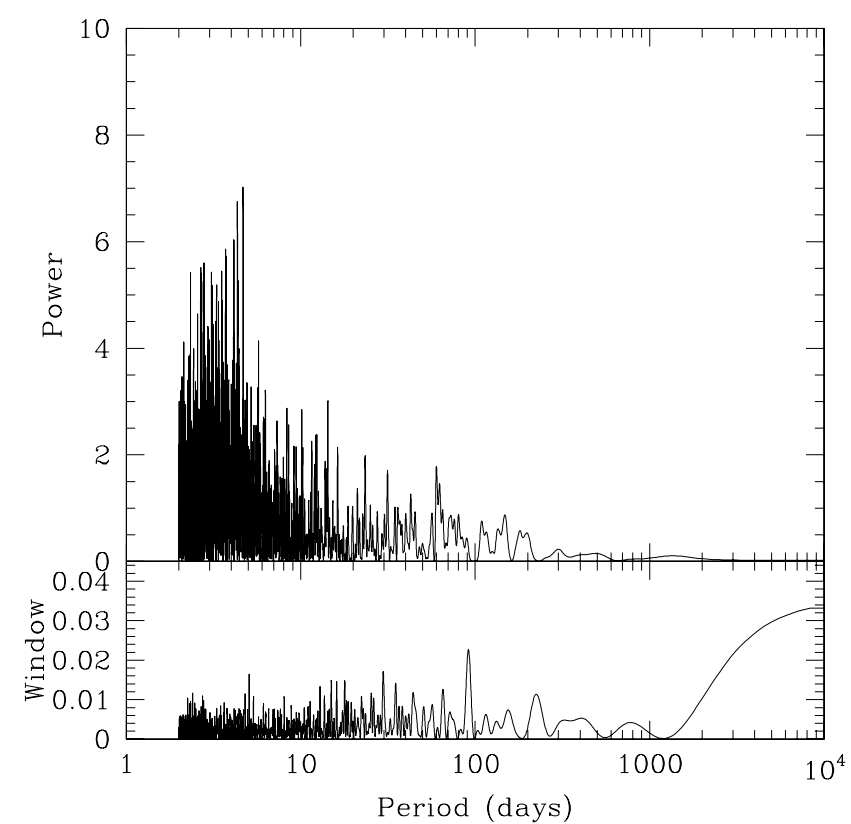

Figure 2. Periodogram of the residuals to the Keplerian orbit fit; no further signals are evident.

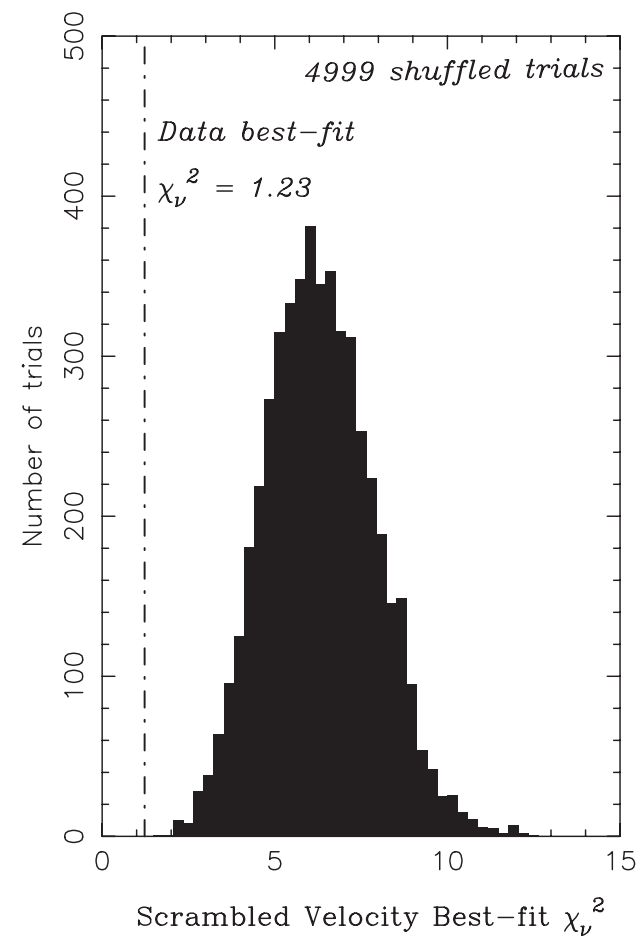

Figure 3. Results of 4999 trials in which the velocity data for $7 \mathrm{CMa}$ were scrambled among the observation epochs. The reduced $\chi^{2}$ of the original data is shown as a dashed vertical line. None of the scrambled data sets resulted in a better $\chi^{2}$, indicating a less than $0.02 \%$ chance that the observed variations are due to noise.

we used the "scrambled velocity" approach of Marcy et al. (2005). This technique serves to test the null hypothesis that the observed velocity variation is attributable to noise. For this test, we scramble the velocities among the observation epochs, creating 5000 shuffled data sets. Then, we perform the same least-squares Keplerian orbit-fitting on the shuffled data and log the resulting best-fit $\chi^{2}$. The results of these trials are shown in Figure 3-not one of the scrambled data sets achieved a better $\chi^{2}$ than the planet fit to our original data. We thus conclude that

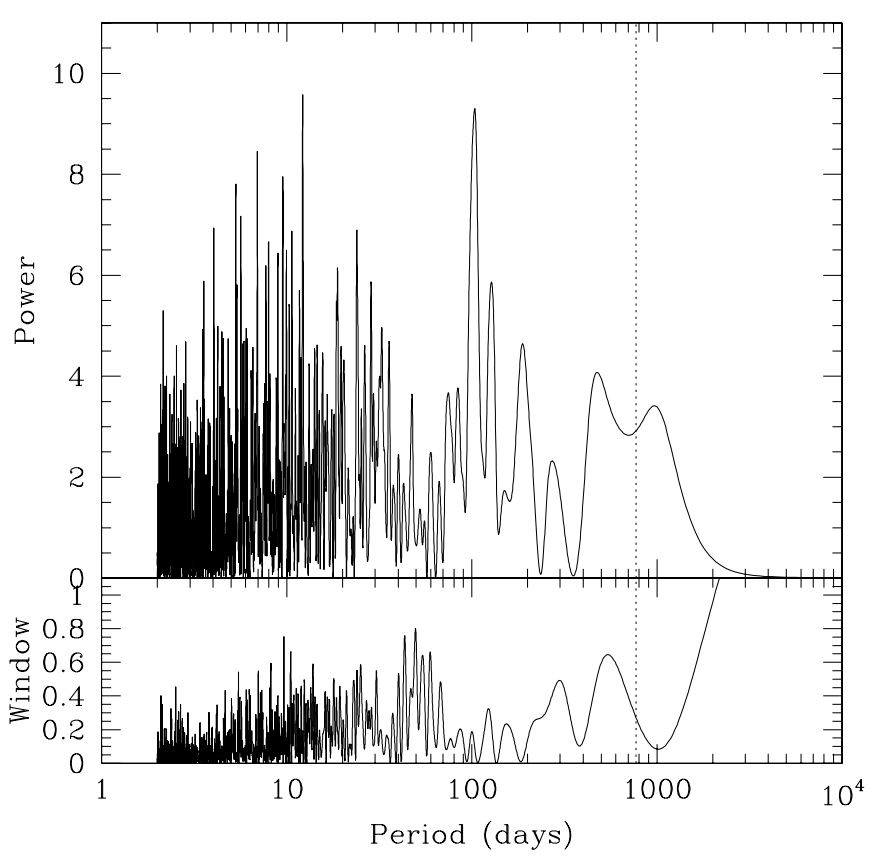

Figure 4. Periodogram of Hipparcos photometry for $7 \mathrm{CMa}(N=168)$. The two highest peaks are at periods of 12.2 and 103.7 days. The vertical dashed line indicates the 763 day period of the planet; there is no significant periodicity in the photometry near this period.

Table 4

7 CMa Planetary Parameters

\begin{tabular}{lc}
\hline \hline Parameter & Estimate \\
\hline Period (days) & $763 \pm 17$ \\
Eccentricity & $0.14 \pm 0.06$ \\
$\omega(\mathrm{deg})$ & $12 \pm 41$ \\
$K\left(\mathrm{~m} \mathrm{~s}^{-1}\right)$ & $44.9 \pm 4.0$ \\
$T_{0}(\mathrm{JD}-2400000)$ & $55520 \pm 89$ \\
$M \sin i\left(M_{\mathrm{Jup}}\right)$ & $2.6 \pm 0.6$ \\
$a(\mathrm{AU})$ & $1.9 \pm 0.1$ \\
\hline $\mathrm{rms}$ of fit $\left(\mathrm{m} \mathrm{s}^{-1}\right)$ & 7.5 \\
$N$ & 21 \\
\hline
\end{tabular}

there is a less than $0.02 \%$ probability that the detected signal arose by chance from noise.

\section{DISCUSSION}

\subsection{Testing the Planet Hypothesis}

Since many $K$ giants have intrinsic radial-velocity variations with periods of hundreds of days (Hekker et al. 2008), it is prudent for us to further examine the planet hypothesis for $7 \mathrm{CMa}$ to ensure that the observed velocity variations are not associated with known activity and rotational cycles. The first and simplest test is to combine the available estimates of the star's radius and $v \sin i$ minimum rotational velocity to obtain a maximum rotation period. Using the values for these quantities given in Table 2, this yields a maximum $P_{\text {rot }}=116$ days (for $v \sin i=1.0 \mathrm{~km} \mathrm{~s}^{-1}$; Massarotti et al. 2008). Unfortunately, neither estimate of $v \sin i$ has an uncertainty, but if we apply a typical uncertainty of $1 \mathrm{~km} \mathrm{~s}^{-1}$, then maximum rotation periods shorter than 776 days fall within the $1 \sigma$ range.

For a spotted star, the rotation period can be deduced from photometry. A periodogram of the Hipparcos photometry (after removing one outlier which was more than 1 mag discrepant) is shown in Figure 4. Two peaks are evident at periods of 

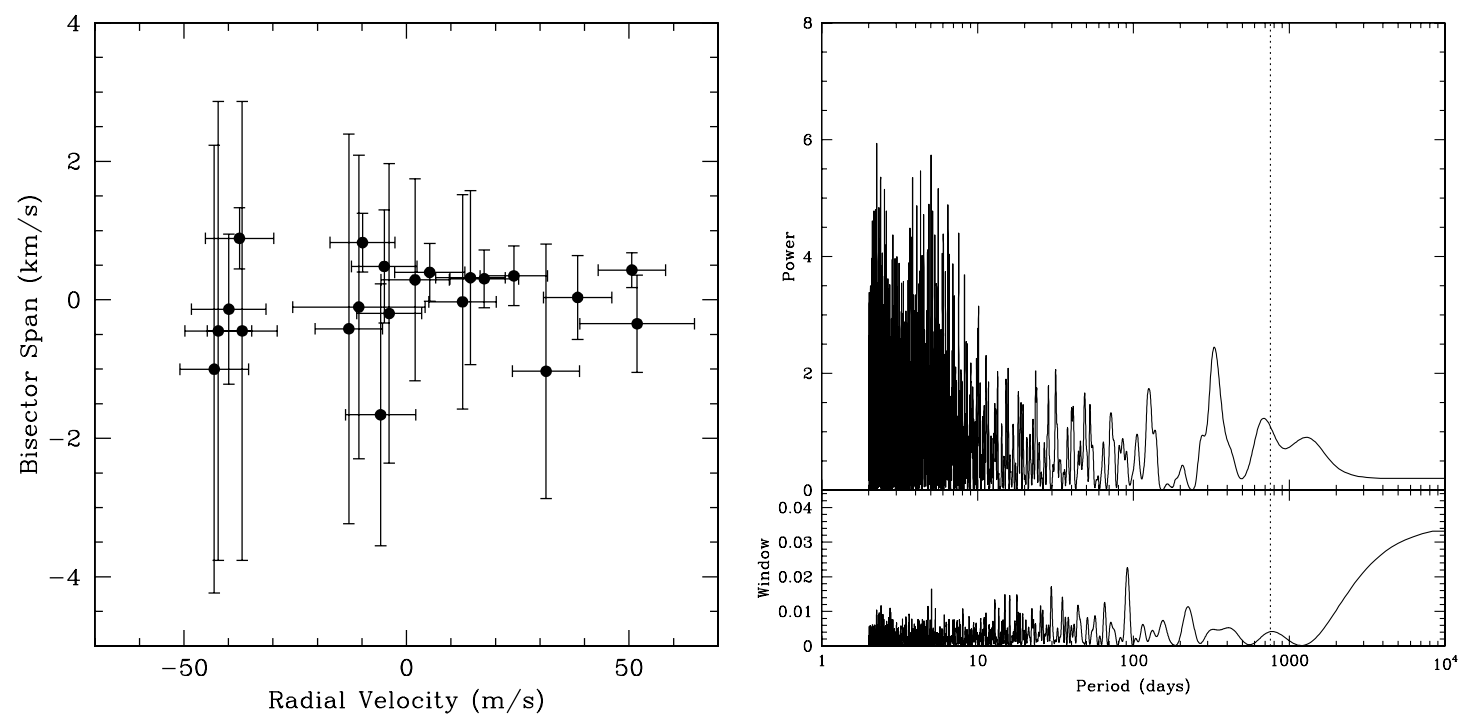

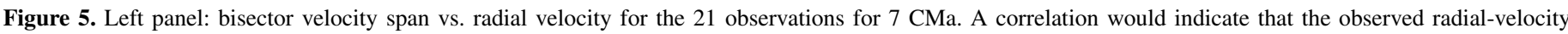

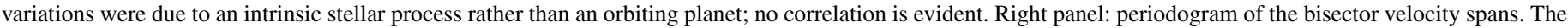
vertical dashed line indicates the 763 day period of the planet; there is no significant periodicity near this period.

12.2 and 103.7 days. We estimate the false alarm probability using the bootstrap randomization method (Kürster et al. 1997). The bootstrap method randomly shuffles the observations while keeping the times of observation fixed. The periodogram of this shuffled data set is then computed and its highest peak recorded. From 10,000 such realizations, we find a false alarm probability of $2.5 \%$ for the peak at 12.2 days, and $3.2 \%$ for that at 103.7 days. At the 763 day period of the candidate planet, the bootstrap false alarm probability is $98.7 \%$. The amplitude of the photometric variations for either of the two marginally significant periods is $20 \pm 6 \times 10^{-4} \mathrm{mag}$. In any case, if these small photometric variations are due to the star's rotation, their periodicities are clearly well separated from that of the candidate planet.

One can argue that the absence of significant variations in the Hipparcos photometry on the 763 day period is not a complete refutation of the starspot hypothesis. Stars have activity cycles, and so there is the possibility that the activity of $7 \mathrm{CMa}$ was at a minimum during the Hipparcos observations (20 years before the radial-velocity observations), but is now at a maximum which, if the rotation period were as long as $\sim 763$ days, could mimic the signal of an orbiting planet. Line bisector analysis is a fairly common technique used by some planet-search programs (e.g., HARPS) to make sure a signal is not due to stellar activity. Such analysis has the advantage of being contemporaneous with the velocity measurements. We note that for the radialvelocity programs using AAT/UCLES, spectra are of relatively low resolution $(R=45,000)$ and nearly all of the usable spectral range is superimposed with iodine lines. We have computed the bisector velocity spans for eight strong unblended lines redward of the iodine region. The results are shown in Figure 5; each point represents the mean bisector velocity span of eight lines, and its uncertainty is the standard deviation about the mean value. While the uncertainties are large, it is evident from Figure 5 that the bisector velocity spans are uncorrelated with the radial velocities. Furthermore, the right panel of Figure 5 shows a periodogram of the bisector velocity spans, which also indicates no periodicity near the 763 day period of the planet. These independent lines of evidence thus lead us to conclude that the radial-velocity variations observed in $7 \mathrm{CMa}$ are attributable not to an intrinsic stellar process, but to an orbiting giant planet.

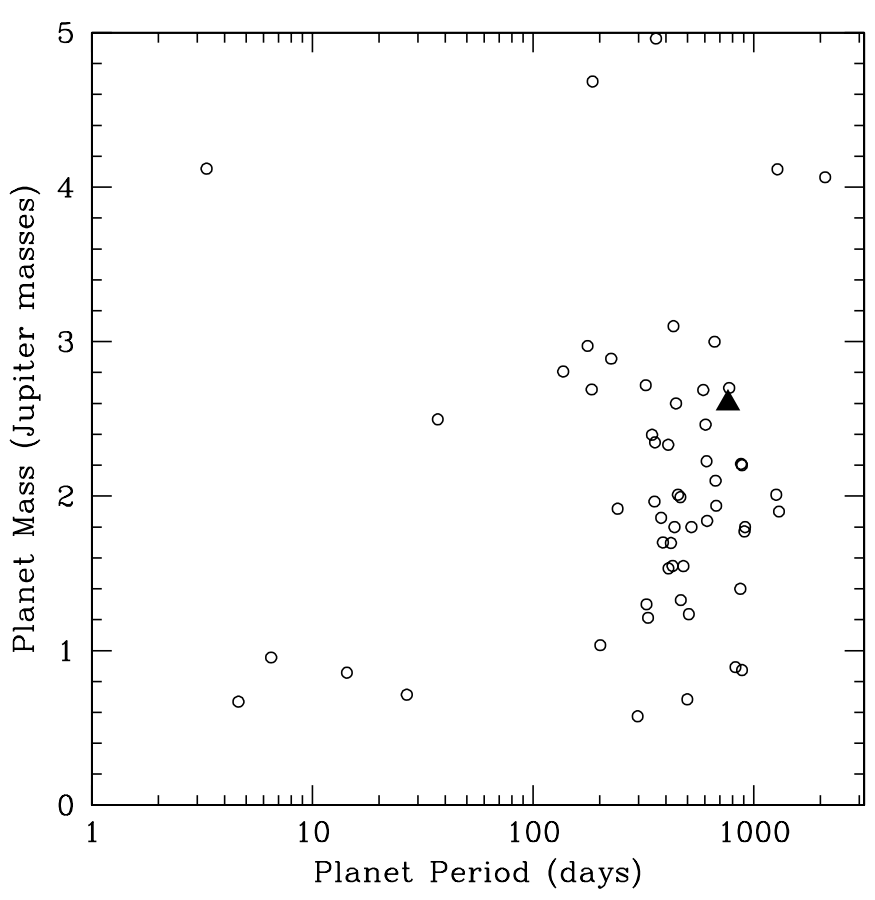

Figure 6. Mass-period plot for 83 radial-velocity-detected planets orbiting stars with $M_{*}>1.3 M_{\odot}$; planet data are from the Exoplanet Orbit Database (Wright et al. 2011). $7 \mathrm{CMa} \mathrm{b}$ is marked as a large filled triangle. Its parameters are consistent with other planets orbiting intermediate-mass stars.

\subsection{Conclusions}

Using the AAT, we have begun a Southern hemisphere search for planets orbiting evolved, intermediate-mass stars. Our PPPS team members are based in Australia, China, and the US; we now report the first planet detection from our ongoing survey. There is an emerging trend that planets orbiting intermediatemass stars tend to have higher masses and longer periods that planets orbiting solar-mass stars (Bowler et al. 2010; Johnson et al. 2010c). With a period of 2.1 years and a minimum mass of $2.6 M_{\mathrm{Jup}}, 7 \mathrm{CMa} \mathrm{b}$ is quite similar to other planets known to orbit intermediate-mass stars. Figure 6 shows the mass and 
period distribution of all radial-velocity-detected planets known to orbit stars with $M_{*}>1.3 M_{\odot}$, with $7 \mathrm{CMa} b$ plotted as a large filled triangle. Given the abundance of planets with $P \gtrsim 2 \mathrm{yr}$ known to orbit these types of stars, we anticipate that $7 \mathrm{CMa} b$ is the first of many planet detections to come from the PPPS.

We gratefully acknowledge the UK and Australian government support of the Anglo-Australian Telescope through their PPARC, STFC, and DIISR funding; STFC grant PP/C000552/ 1, ARC Grant DP0774000, and travel support from the Australian Astronomical Observatory. R.W. is grateful to the Chinese Academy of Sciences for the support of his stay in Beijing. R.W. is supported by a UNSW Vice-Chancellor's Fellowship.

We thank the ATAC for the generous allocation of telescope time which facilitated this detection. This research has made use of NASA's Astrophysics Data System (ADS), and the SIMBAD database, operated at CDS, Strasbourg, France. This research has made use of the Exoplanet Orbit Database and the Exoplanet Data Explorer at exoplanets.org.

\section{REFERENCES}

Bowler, B. P., Johnson, J. A., Marcy, G. W., et al. 2010, ApJ, 709, 396 Butler, R. P., Marcy, G. W., Williams, E., et al. 1996, PASP, 108, 500 Charbonneau, P. 1995, ApJS, 101, 309

Cochran, W. D., Endl, M., Wittenmyer, R. A., \& Bean, J. L. 2007, ApJ, 665 , 1407

da Silva, L., Girardi, L., Pasquini, L., et al. 2006, A\&A, 458, 609

Diego, F., Charalambous, A., Fish, A. C., \& Walker, D. D. 1990, Proc. SPIE, 1235,562

Döllinger, M. P., Hatzes, A. P., Pasquini, L., et al. 2007, A\&A, 472, 649

Endl, M., Cochran, W. D., Kürster, M., et al. 2006, ApJ, 649, 436

Endl, M., Hatzes, A. P., Cochran, W. D., et al. 2004, ApJ, 611, 1121

Endl, M., Kürster, M., \& Els, S. 2000, A\&A, 362, 585

Fischer, D. A., \& Valenti, J. 2005, ApJ, 622, 1102

Galland, F., Lagrange, A.-M., Udry, S., et al. 2005, A\&A, 443, 337

Girardi, L., Bertelli, G., Bressan, A., et al. 2002, A\&A, 391, 195

Gonzalez, G. 1999, MNRAS, 308, 447

Gray, R. O., Corbally, C. J., Garrison, R. F., et al. 2006, AJ, 132, 161

Hatzes, A. P., Guenther, E. W., Endl, M., et al. 2005, A\&A, 437, 743

Hekker, S., \& Meléndez, J. 2007, A\&A, 475, 1003
Hekker, S., Reffert, S., Quirrenbach, A., et al. 2006, A\&A, 454, 943 Hekker, S., Snellen, I. A. G., Aerts, C., et al. 2008, A\&A, 480, 215 Howard, A. W., Marcy, G. W., Johnson, J. A., et al. 2010, Science, 330, 653 Jefferys, W. H., Fitzpatrick, M. J., \& McArthur, B. E. 1987, Celest. Mech., 41, 39

Johnson, J. A. 2008, in ASP Conf. Ser. 398, Extreme Solar Systems, ed. D. Fischer, F. A. Rasio, S. E. Thorsett, \& A. Wolszczan (San Francisco, CA: ASP), 59

Johnson, J. A., Aller, K. M., Howard, A. W., \& Crepp, J. R. 2010a, PASP, 122, 905

Johnson, J. A., Bowler, B. P., Howard, A. W., et al. 2010b, ApJ, 721, L153

Johnson, J. A., Fischer, D. A., Marcy, G. W., et al. 2007, ApJ, 665, 785

Johnson, J. A., Howard, A. W., Bowler, B. P., et al. 2010c, PASP, 122, 701

Johnson, J. A., Marcy, G. W., Fischer, D. A., et al. 2006a, ApJ, 647, 600

Johnson, J. A., Marcy, G. W., Fischer, D. A., et al. 2006b, ApJ, 652, 1724

Johnson, J. A., Payne, M., Howard, A. W., et al. 2011, AJ, 141, 16

Kürster, M., Schmitt, J. H. M. M., Cutispoto, G., \& Dennerl, K. 1997, A\&A, 320,831

Marcy, G. W., Butler, R. P., Vogt, S. S., et al. 2005, ApJ, 619, 570

Massarotti, A., Latham, D. W., Stefanik, R. P., \& Fogel, J. 2008, AJ, 135, 209

Murray, N., \& Chaboyer, B. 2002, ApJ, 566, 442

Niedzielski, A., Goździewski, K., Wolszczan, A., et al. 2009, ApJ, 693, 276

O’Toole, S., Jones, H. R. A., Tinney, C. G., et al. 2009, ApJ, 701, 1732

O’Toole, S., Tinney, C. G., Butler, R. P., et al. 2009a, ApJ, 697, 1263

Pasquini, L., Döllinger, M. P., Weiss, A., et al. 2007, A\&A, 473, 979

Perryman, M. A. C., Lindegren, L., Kovalevsky, J., et al. 1997, A\&A, 323, L49

Saar, S. H., Butler, R. P., \& Marcy, G. W. 1998, ApJ, 498, L153

Sandage, A., Lubin, L. M., \& VandenBerg, D. A. 2003, PASP, 115, 1187

Sato, B., Kambe, E., Takeda, Y., et al. 2005, PASJ, 57, 97

Sato, B., Omiya, M., Liu, Y., et al. 2010, PASJ, 62, 1063

Schuler, S. C., Kim, J. H., Tinker, M. C., Jr., et al. 2005, ApJ, 632, L131

Setiawan, J., Pasquini, L., da Silva, L., von der Lühe, O., \& Hatzes, A. 2003, A\&A, 397, 1151

Takeda, G., Ford, E. B., Sills, A., et al. 2007, ApJS, 168, 297

Tinney, C. G., Wittenmyer, R. A., Butler, R. P., et al. 2011, ApJ, 732, 31

Valenti, J. A., Butler, R. P., \& Marcy, G. W. 1995, PASP, 107, 966

Valenti, J. A., \& Fischer, D. A. 2005, ApJS, 159, 141

Valenti, J. A., \& Fischer, D. A. 2008, Phys. Scr. T, 130, 014003

van Leeuwen, F. 2007, A\&A, 474, 653

Vogt, S. S., Wittenmyer, R. A., Butler, R. P., et al. 2010, ApJ, 708, 1366

Wang, L., Liu, Y., Zhao, G., \& Sato, B. 2011, PASJ, 63, 1035

Wittenmyer, R. A., Endl, M., Cochran, W. D., Levison, H. F., \& Henry, G. W. 2009, ApJS, 182, 97

Wittenmyer, R. A., Tinney, C. G., Butler, R. P., et al. 2011, ApJ, 738, 81

Wright, J. T. 2005, PASP, 117, 657

Wright, J. T., Fakhouri, O., Marcy, G. W., et al. 2011, PASP, 123, 412 